\title{
Enfermedad obstructiva crónica (EPOC) y comorbilidades
}

\section{Chronic obstructive pulmonary disease and comorbidities}

\author{
Fernando de la Iglesia Martínez ${ }^{1}$, Joaquín Serrano Arreba', Julio Montes Santiago². \\ ${ }^{1}$ Servicio de Medicina interna. Complejo Hospitalario Universitario A Coruña (CHUAC). SERGAS. A Coruña \\ ${ }^{2}$ Servicio de Medicina interna. Complejo Hospitalario Universitario Vigo (CHUVI). SERGAS. Vigo
}

\section{Resumen}

Las agudizaciones de la EPOC y la insuficiencia cardiaca (IC) constituyen la principal causa de hospitalización en los servicios de Medicina interna en España. Además ambas entidades frecuentemente coexisten. La EPOC es una entidad que muy habitualmente trasciende el ámbito pulmonar y se asocia con un elevado número de enfermedades que afectan a distintos órganos. Ente ellas están la cardiopatía isquémica, la enfermedad cerebrovascular, las infecciones pulmonares recidivantes, etc. En tal situación tiene un papel relevante el tabaco como principal factor de riesgo para desarrollar EPOC y también agente etiológico principal en otros procesos y también por la capacidad del mismo como inductor de reacciones inflamatorias, que hace que la EPOC comparta muchas características comunes con gran variedad de procesos sistémicos. No obstante, tal agente no explica totalmente la incidencia incrementada en la EPOC de otras entidades como la depresión o la diabetes mellitus. En cualquier caso la presencia de EPOC con otros procesos comórbidos incrementa la mortalidad y dificulta el manejo asistencial.

PALABRAS CLAVES: Enfermedad pulmonar obstructiva crónica. Tabaco. Pacientes pluripatológicos.

\section{Epidemiologia de la EPOC y entidades asociadas}

Según estimaciones de la OMS (2007), actualmente unos 210 millones de personas sufren EPOC'1. En 2005, 3 millones fallecieron por esta causa, lo cual representa el $5 \%$ del global de mortalidad. Su impacto sanitario, social y económico es muy elevado. Se estima que en el 2020 la EPOC se habrá convertido en la $3^{\mathrm{a}}$ causa de muerte en todo el mundo ${ }^{2}$. En 2004 se estima en 30,2 millones las pérdidas por Años de Vida Ajustados por Discapacidad (AVAD). Esta cifra representa el 2,0\% sobre el total de AVAD. Confirmando estas cifras, una reciente revisión de más de 60 estudios poblacionales estiman dicha prevalencia en población general en cerca del $1 \%$, creciendo hasta el 8-10\% o más en adultos $>40$ años ${ }^{3}$.

\begin{abstract}
The exacerbations of Chronic Obstructive Pulmonary Disease (COPD) and Heart failure (HF) are leading causes of hospitalization in the services of Internal Medicine in Spain. Moreover, both entities often coexist. COPD is an entity which frequently transcends the lung and is associated with a large number of diseases affecting different organs. Among these ischemic heart disease, cerebrovascular disease, recurrent pulmonary infections, etc. In such situations tobacco play an important role as the main risk factor for developing COPD and as the etiologic factor in other processes. The ability of tobacco to induce inflammatory reactions makes COPD share many common features with a variety of systemic process. However, such an agent does not fully explain the increased incidence in COPD of the other entities like depression or diabetes mellitus. In any case, the presence of COPD in addition to other comorbidities increases mortality and difficult to manage these patients.
\end{abstract}

KEYWORDS: Chronic obstructive pulmonary disease. Tobacco. Patients with Plurypathology.

La EPOC también es una causa de elevada morbilidad, mortalidad y discapacidad en España. Se estima la prevalencia de la EPOC en nuestro medio entre un 6,4 y un 11,4\%, según las zonas geográficas ${ }^{4}$. El estudio IBERPOC 5 (1998) encontró un $9,1 \%$ (con $78 \%$ de casos no diagnosticados) y el estudio

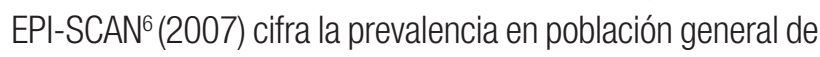
40-79 años en 10,2\% (con 73\% no diagnosticados). En este último estudio la prevalencia en la zona de Vigo fue del 8,2\%. Según la Encuesta de Morbilidad Hospitalaria (2005) las altas por EPOC supusieron el 6,5\% del total ${ }^{4}$. En España las enfermedades respiratorias suponen la $4^{\text {a }}$ causa de carga de enfermedad (7,5\% del total de AVAD) con un componente importante de discapacidad ${ }^{4}$. En hombres, la EPOC se encuentra entre las enfermedades con un mayor número

Tabla 1. Características de los pacientes con IC, EPOC y ambos procesos en el SNS (2009)

\begin{tabular}{|l|c|c|c|}
\hline \multicolumn{1}{|c|}{2009} & IC & EPOC & IC + EPOC (2005) \\
\hline No > 35 años & 88.756 & 71.047 & 30.139 \\
\hline Mujeres (\%) & 54 & 19 & 42 \\
\hline$>75$ años (\%) & 72 & 55 & 76,3 \\
\hline Edad media al ingreso & 78,0 & 73,3 & 9,2 \\
\hline Estancia media & 9,2 & 8,6 & 9,8 \\
\hline Mortalidad (\%) & 10,0 & 4,4 & 4.108 \\
\hline Coste medio () & 4.243 & 2.661 & \\
\hline
\end{tabular}




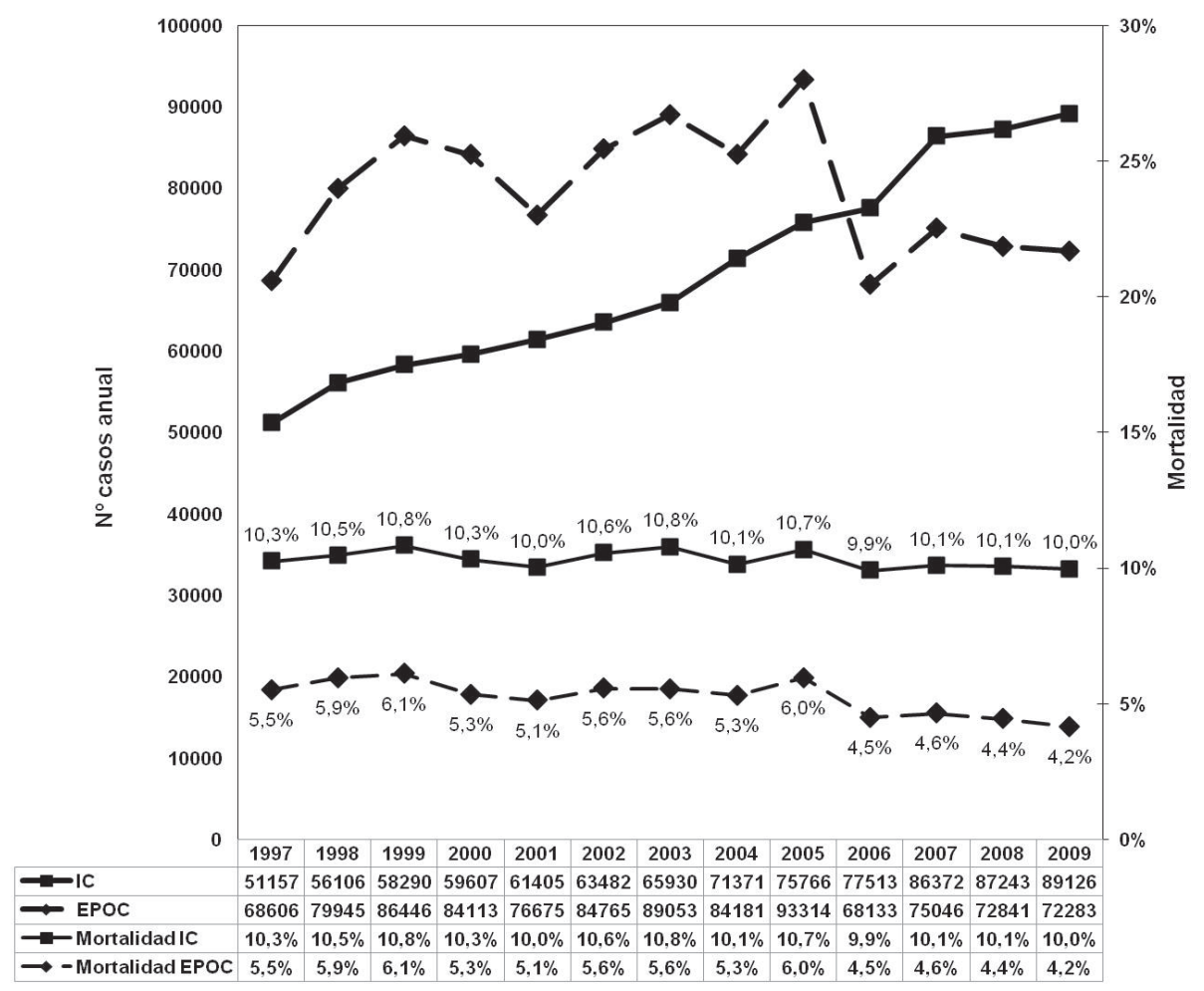

de AVAD (4,1\% sobre el total de AVAD), siendo en mujeres la carga de enfermedad algo inferior (1,3\% sobre el total de AVAD) ${ }^{4}$. Globalmente, los gastos totales asociados a la EPOC equivalen al $0,2 \%$ del PIB español ${ }^{4}$. Ello además puede ser mucho mayor ya que se estima en cerca del $75 \%$ los casos no diagnosticados. Contando estos se calcula que en España existirían aproximadamente unos 270.000 pacientes con EPOC diagnosticados y tratados, lo cual se traduciría en cerca de 473 millones anuales sólo en gastos sanitarios directos generados por la EPOC ${ }^{4}$. En cuanto a su distribución se observa que los gastos de hospitalización son los más cuantiosos (40-45\% de los costos), seguidos del farmacológico (35-40\%) y las visitas y pruebas diagnósticas (15-25\%)

Con respecto a la mortalidad en 2005 en España la EPOC fue la 5 a causa entre los varones (Tasa anual 61/100.000 h.) y la $7^{\mathrm{a}}$ en mujeres (20/ 100.000 h.), siendo la edad media de muerte de 79,7 en varones y 83,7 en mujeres ${ }^{4}$. En ese año los años de vida perdidos (por 100000) fueron de 62,3 en hombres y 17,7 en mujeres ${ }^{4}$.

Por su parte, la insuficiencia cardiaca (IC) presenta una prevalencia del $6,8 \%$ de la población $>45$ años (2005) aunque esta sube acusadamente en edades más avanzadas (16,1\% en $>75$ años $)^{8}$. Ello hace que en España se produzcan anualmente $>100000$ hospitalizaciones, con un gasto calculado de estas de 430 millones, y existan cerca de 1,25 millones de personas con tal entidad ${ }^{9}$. Aunque se tienen menos datos sobre la coexistencia de ambos procesos, estudios en pacientes hospitalizados primariamente por IC en el Sistema Nacional de salud (SNS) señalan que el 34\% de los mismos presenta EPOC concomitante ${ }^{10}$. En estudios de Registros de pacientes con IC en Medicina Interna (SEMI-IC, RICA) esta prevalencia de EPOC oscila entre el 28-31\% ${ }^{11-12}$. A la inversa, en Registros de pacientes con EPOC la prevalencia de IC es de un $27 \%{ }^{13}$. En el SNS las características entre ambos grupos de pacientes se aprecian en la tabla 1.

Ambas patologías (IC y EPOC) constituyen la causa más frecuente de ingresos en los Servicios de Medicina Interna del SNS, determinando entre ambas más de 120.000 hospitalizaciones anuales en dichos Servicios y constituyendo el 26,4\% del total de hospitalizaciones en los mismos ${ }^{14}$. Además se espera que los ingresos por ambas entidades seguirán incrementándose en años venideros, constituyendo un problema sanitario y económico de preocupante magnitud (fig. 1). Con respecto a su mortalidad se observa cierta disminución en los pacientes con EPOC, pero no así en IC que ha permanecido estable (fig. 1). Además la mayoría de estos pacientes presentan el perfil de añosos (25\% de pacientes con EPOC tienen $>80$ años) $)^{13}$ y con pluripatología. Así las características de pacientes con EPOC hospitalizados en Servicios de Medicina Interna son una edad media de 74 años, con hipertensión arterial (55\%), arritmias (27\%), insuficiencia cardiaca (27\%) y diabetes mellitus (26\%), con una media de cerca de 4 enfermedades asociadas ${ }^{15}$.

\section{La EPOC como enfermedad sistémica.}

La actualización de 2011 de la Global Initiative for Chronic Obstructive Lung Disease (GOLD) define a la EPOC como 
"una enfermedad prevenible y tratable, caracterizada por una limitación al flujo aéreo persistente que generalmente progresa y asociada a una respuesta inflamatoria crónica aumentada en la vía aérea y en el pulmón a partículas o gases nocivos. Las exacerbaciones y la comorbilidad contribuyen a la gravedad global en los pacientes individuales ${ }^{\prime \prime 6}$. Por lo tanto los expertos de la GOLD incluyen en la propia definición el reconocimiento de las indudables implicaciones que tienen las manifestaciones sistémicas de la EPOC y la comorbilidad en el cuidado de estos pacientes.

La inhalación fundamentalmente de humo de tabaco causa una inflamación en el pulmón que se caracteriza por el aumento de los neutrófilos, macrófagos y linfocitos T, especialmente los $\mathrm{CD} 8^{+17}$. El proceso inflamatorio, el disbalance entre proteinasas/antiproteinasas y el stress oxidativo ${ }^{18}$ conducirán a unos cambios anatómicos (hipersecreción de moco, disfunción ciliar, estrechamiento y fibrosis de la vía aérea, destrucción del parénquima y cambios vasculares) que conducen a la característica limitación al flujo aéreo, así como a la hiperinsuflación pulmonar, anomalías en el intercambio gaseoso, hipertensión pulmonar y efectos sistémicos como datos de inflamación sistémica y disfunción músculoesquelética ${ }^{19}$. Este círculo vicioso condiciona una disminución de la tolerancia al ejercicio, lo que favorece un menor grado de actividad física y condiciona un progresivo deterioro de la forma física que limita la capacidad de ejercicio del paciente y disminuye su estado de salud.

La inflamación sistémica de la EPOC se demuestra por un incremento de leucocitos, proteína C reactiva (PCR), fibrinógeno y citoquinas inflamatorias en la sangre de los pacientes en situación estable 20,21 , lo que traduce un estado de inflamación sistémica persistente de bajo grado (Figura 2). Además, durante las exacerbaciones se elevan los niveles de interleukina-6, PCR, fibrinógeno y factor de necrosis tumoral (TNF-a) que disminuyen de nuevo durante la recuperación ${ }^{20,21}$.

Fig.2. Inflamación sistémica y EPOC.

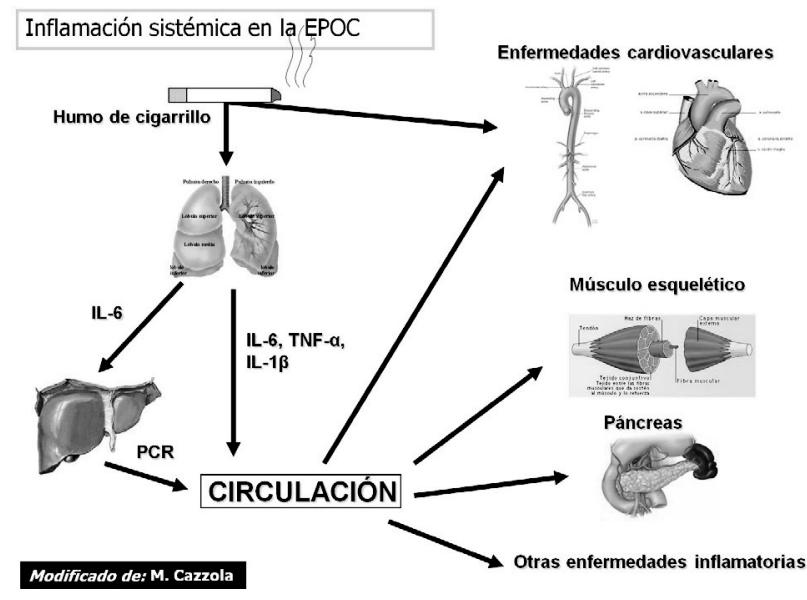

El origen de esta inflamación sistémica puede deberse a diferentes mecanismos:

a) "desbordamiento" de moléculas proinflamatorias desde el parénquima pulmonar inflamado y/o activación de células inflamatorias (neutrófilos, monocitos, linfocitos) durante su paso por la circulación pulmonar;

b) que otros órganos como el hígado, músculo esquelético o médula ósea puedan contribuir a la producción de citoquinas proinflamatorias;

c) que el humo del cigarrillo tenga por sí mismo potencial para producir inflamación sistémica, como lo demuestra la ocurrencia de enfermedad coronaria (también una enfermedad inflamatoria) en fumadores, independientemente de la presencia 0 ausencia de EPOC ${ }^{21}$.

Independientemente de su origen esta inflamación sistémica junto con otros factores como el sedentarismo, la hipoxia tisular, el envejecimiento, la malnutrición y los efectos secundarios de los fármacos conducen a los efectos sistémicos de la EPOC: pérdida de peso, disfunción músculo esquelética, enfermedades cardiovasculares, diabetes o intolerancia a la glucosa, depresión, etc ${ }^{21,22}$.

\section{Comorbilidades en pacientes con EPOC}

El componente inflamatorio extrapulmonar de la EPOC es la base que sustenta el concepto de enfermedad sistémica. Aunque hasta el momento no existe un consenso claro sobre la diferencia entre manifestaciones sistémicas en la EPOC y comorbilidad, algunos autores han sugerido catalogar como manifestaciones sistémicas las alteraciones extrapulmonares directamente relacionadas con la enfermedad, como la pérdida de peso o la miopatía, y reservar el término comorbilidad para aquellas patologías asociadas ${ }^{23}$. La importancia de las patologías asociadas la pone de manifiesto la demostración de que en los pacientes con EPOC, además de la insuficiencia respiratoria, la cardiopatía isquémica y las neoplasias son causas frecuentes de fallecimiento ${ }^{24,25}$.

Para valorar la comorbilidad se utiliza a menudo el índice de Charlson, una escala validada diseñada para valorar el riesgo de muerte y su relación con enfermedades crónicas en estudios longitudinales ${ }^{26}$. Este índice ha demostrado ser un buen predictor pronóstico en la EPOC ${ }^{27}$, aunque su uso exlusivo produce una pérdida de información sobre patologías no incluidas en él y que pueden tener importancia en Ios síntomas y la evolución de la EPOC como la anemia 0 depresión, entre otros ${ }^{21,22}$.

Con la intención de conocer la comorbilidad de estos pacientes el grupo EPOC de la Sociedad Española de Medicina Interna llevó a cabo un estudio multicéntrico, prospectivo y observacional que finalmente incluyó a 398 pacientes que precisaron ingresar en los Servicios de Medicina Interna por 
exacerbación de su EPOC ${ }^{13}$. La mayoría, el 89\%, eran varones, con una edad media de 73,7 años, la estancia media observada fue de 9,8 días y fallecieron en el ingreso el $5 \%{ }^{13}$. El número de enfermedades crónicas asociadas a la EPOC fue de 3,6, mientras que la puntuación media observada para el índice de Charlson fue de 2,72; en la tabla 2 se recogen con detalle las diferentes comorbilidades ${ }^{13}$. La coexistencia de EPOC con otras patologías ha sido reconocida como factor de mal pronóstico ya que dicha asociación, especialmente con enfermedades cardiovasculares, modifica el pronóstico de ambas ${ }^{13}$. Además, es sabido que las pruebas de función respiratoria son un predictor de mortalidad en la población general y especialmente en los pacientes con cardiopatía ${ }^{28}$. Ya sea por la EPOC en sí, por complicaciones de la misma 0 por enfermedades asociadas lo que sabemos es que los pacientes con EPOC tienen una menor esperanza de vida que la población general, como demuestran los datos de un estudio realizado en Gran Bretaña en el que los pacientes con EPOC severo fallecían tres años antes que aquellos con enfermedad moderada y cuatro años antes que los pacientes control con la misma edad y sexo ${ }^{29}$.

\section{EPOC y enfermedad vascular.}

La EPOC comparte con las enfermedades vasculares un factor de riesgo común, el tabaquismo, pero este factor no explica por sí sólo que los pacientes con EPOC tengan entre 2 y 3 veces más riesgo de mortalidad cardiovascular en los grandes estudios poblacionales ${ }^{30-32}$. La investigación actual sugiere que la alteración inflamatoria contribuye al proceso de ateroesclerosis ${ }^{33}$ y puede ser el nexo común en ambos grupos de enfermedades. El incremento en los niveles de PCR representa un factor de riesgo de enfermedad cardiovascular y en pacientes con EPOC se ha demostrado un aumento de dichos niveles y su relación con un mayor número de eventos cardiovasculares posteriores ${ }^{34}$. Esta actividad inflamatoria podría explicarse porque la producción de citoquinas inflamatorias en el pulmón conduzca a un estado de hipercoagulabilidad predisponente a trombosis y predisponga también a la ruptura de la placa de ateroma ${ }^{33}$.

La asociación de cardiopatía isquémica en el paciente EPOC es muy frecuente en el mundo occidental. El estudio ECCO confirmó la presencia de enfermedad coronaria en un 17\% de los pacientes y hasta en un 9\% existía el antecedente de infarto de miocardio previo ${ }^{13}$. Ambas enfermedades comparten el tabaquismo como factor predisponerte y predilección por la población de mayor edad. La afectación de personas de mayor edad requiere por parte del clínico un mayor conocimiento de la potenciación de ambas enfermedades y de las posibles interacciones medicamentosas en su tratamiento conjunto. Como regla general los pacientes con ambas patologías deben realizar tratamiento para ambas como si existieran de
Tabla 2. Frecuencia de las comorbilidades en EPOC (Estudio ECCO, $\mathrm{n}=398)$ (modif. de ref. $\left.{ }^{13}\right)$

\begin{tabular}{|l|c|}
\hline \multicolumn{1}{|c|}{ Comorbilidad } & Total \\
\hline Incluidas en el índice de Charlson & \\
\hline Enfermedad coronaria & $68(17 \%)$ \\
\hline Insuficiencia cardíaca & $107(27 \%)$ \\
\hline Enfermedad vascular periférica & $50(13 \%)$ \\
\hline Enfermedad cerebrovascular & $38(10 \%)$ \\
\hline Demencia & $15(4,4 \%)$ \\
\hline Enfermedad del tejido conectivo & $7(2 \%)$ \\
\hline Ulcus péptico & $49(12 \%)$ \\
\hline Hepatopatía leve & $29(7,3 \%)$ \\
\hline Diabetes sin complicaciones & $103(26 \%)$ \\
\hline Diabetes con daño orgánico & $14(3,5 \%)$ \\
\hline Hemiplejia & $4(1 \%)$ \\
\hline Insuficiencia renal moderada & $26(6,5 \%)$ \\
\hline Tumor sólido sin metástasis & $26(6,5 \%)$ \\
\hline Leucemia & $6(1,5 \%)$ \\
\hline Linfoma & $2(0,5 \%)$ \\
\hline Enfermedad hepática moderada 0 severa & $9(2,3 \%)$ \\
\hline Tumor sólido con metástasis & $7(1,8 \%)$ \\
\hline Síndrome de inmunodeficiencia adquirida & $1(0,3 \%)$ \\
\hline Otras comorbilidades & $34(9,7 \%)$ \\
\hline Infarto de miocardio & $218(55 \%)$ \\
\hline Hipertensión arterial & $56(14 \%)$ \\
\hline Alcoholismo & $13(3 \%)$ \\
\hline Enfermedad tromboembólica & \\
\hline Arritmia & $27 \%)$ \\
\hline Edemas & $32 \%)$ \\
\hline Osteoporosis & \\
\hline Anemia & \\
\hline
\end{tabular}

forma independiente con algunas precauciones. Si se inicia tratamiento con betabloqueantes se deben elegir los cardioselectivos, en dosis bajas y con progresión gradual ${ }^{35}$. Si son bien tolerados se deben mantener también en las agudizaciones.

Los calcioantagonistas y los nitratos no presentan ninguna contraindicación en la EPOC. Los salicilatos se deben usar con precaución en los que presenten hiperreactividad bronquial, sustituyéndose, si es posible, por otro antiagregante no salicílico.

Los esteroides, anticolinérgicos y leucotrienos usados en el tratamiento de la EPOC no modifican el curso de la cardiopatía. No parece que los beta-2-agonistas de corta duración tengan efecto en un paciente que no presente mayor hipoxemia de la habitual, y su discontinuación podría favorecer la aparición de angina por empeoramiento de la oxigenación 
tisular. Lo que sí se recomienda, aunque no existen estudios diseñados específicamente, es evitar dosis altas de betaagonistas en situación de angina inestable ${ }^{16}$.

La prevalencia de insuficiencia cardíaca en el estudio ECCO, de un $27 \%$, es similar a la descrita en pacientes ambulatorios con EPOC grave a los que se le realiza un ecocardiograma ${ }^{13,36}$; esta proporción puede llegar al 50\% en los EPOC graves ingresados en Cuidados Intensivos. La coexistencia de ambas enfermedades disminuye la capacidad de esfuerzo de los pacientes y genera dependencia funcional ${ }^{36}$. Además la disnea o fatiga es síntoma cardinal en ambas entidades, y en ocasiones es difícil conocer qué componente predomina en una exacerbación de estos pacientes. En estos casos, y sin contar con ecocardiografía, puede ser útil la determinación de péptido natriurético cerebral (BNP), ya que valores superiores a 500-1000 pg/ml apoyarían la presencia de insuficiencia cardíaca en estos enfermos ${ }^{13,16}$

El tratamiento de la insuficiencia cardíaca en pacientes con EPOC debe seguir básicamente las recomendaciones generales de las guías. Como en el caso de la enfermedad coronaria los beta-bloqueantes cardioselectivos son los preferibles y su beneficio claramente supera cualquier riesgo potencial asociado al tratamiento, incluso en pacientes con EPOC severa $^{16}$. La incidencia de efectos secundarios de los IECAs en pacientes con asma o EPOC es similar a la de la población sana, por lo que deben usarse siempre que estén indicados. Aunque el uso de beta-2-agonistas se ha relacionado con un aumento de la incidencia de insuficiencia cardíaca, arritmias cardíacas, cardiopatía isquémica y muerte súbita, no existe una evidencia suficiente para contraindicar su uso, si bien parece razonable realizar un seguimiento más cercano a los pacientes con insuficiencia cardíaca severa que estén con este tratamiento para la EPOC ${ }^{16}$.

El 10\% de los pacientes analizados en el estudio ECCO presentaba el antecedente de ictus ${ }^{13}$. La relación entre EPOC y enfermedad cerebrovascular está demostrada en estudios que confirman una relación inversa entre la reducción del FEV1, la incidencia de accidentes cerebrovasculares y el riesgo de ictus mortal ${ }^{37,38}$. El mecanismo exacto por el que la incidencia y la mortalidad de ictus en pacientes con EPOC es mayor es desconocido, si bien la inflamación sistémica demostrada en la EPOC puede desencadenar y perpetuar el proceso aterosclerótico y producir de forma secundaria la oclusión de los vasos cerebrales. Algunos factores han sido identificados, como el tabaquismo, la susceptibilidad para las infecciones, la hipoxemia crónica o la hipercapnia, y la comorbilidad asociada como HTA, fibrilación auricular, insuficiencia cardíaca y enfermedad coronaria. También se considera que algunos fármacos como los simpaticomiméticos y anticolinérgicos pueden aumentar la frecuencia cardíaca y la presión arterial, desencadenar arritmias y por todo ello aumentar el riesgo de accidentes isquémicos transitorios 0 ictus $^{30}$. La asociación de ambas enfermedades en un mismo paciente es motivo de importantes alteraciones físicas, invalidez y consumo de recursos sanitarios y sociales.

Aunque no existen datos precisos se estima que la prevalencia de enfermedad tromboembólica venosa en pacientes hospitalizados por EPOC oscila entre el 8 y 25\%39,40. Además de ser la EPOC en sí misma una situación protrombótica, otros factores como la reducción de la actividad física deben jugar un papel en este hecho. La enfermedad tromboembólica se ha relacionado además con las agudizaciones de la EPOC. En un estudio prospectivo se diagnosticó embolia de pulmón en uno de cada cuatro pacientes ingresados por exacerbación de EPOC sin causa clara de descompensación (infección respiratoria, neumotórax , ...) o bien que presentaban discrepancias entre clínica y hallazgos radiológicos ${ }^{40}$. Por todo ello se debe indicar profilaxis con heparina de bajo peso molecular en los pacientes ingresados por descompensación grave de la EPOC e investigar la posibilidad de embolia pulmonar cuando la causa de la agudización no sea aparente.

\section{EPOC e infección}

Desde un punto de vista teórico es razonable considerar que los pacientes con EPOC tienen un riesgo aumentado para contraer y desarrollar infecciones por varios motivos: a) las alteraciones funcionales y estructurales de la propia enfermedad reducen la capacidad defensiva del pulmón, b) el consumo de tabaco favorece las infecciones respiratorias, c) algunos tratamientos habituales de la EPOC, como los esteroides y los antibióticos alteran la flora microbiana ${ }^{41}, y$ d) los factores asociados a la habitual edad avanzada de los pacientes con EPOC y a la coexistencia de otros procesos crónicos. La experiencia confirma mayores cifras de enfermedades infecciosas en pacientes con EPOC de reciente diagnóstico comparados con una población sin EPOC durante 1 año, siendo éstas el segundo factor de comorbilidad más frecuente, sólo superadas por las cardíacas ${ }^{42}$.

En condiciones normales las vías aéreas son estériles; sin embargo, entre un 25 y $50 \%$ de los pacientes con EPOC en fase estable se encuentran colonizados con los mismos gérmenes que provocan las infecciones respiratorias, siendo más probable dicha colonización cuanto mayor sea el deterioro de la función pulmonar ${ }^{43}$. Esta colonización del árbol bronquial es el principal factor de riesgo para el desarrollo de agudizaciones con episodios de incremento de expresividad clínica de la enfermedad que interrumpen el curso crónico y lentamente progresivo de la EPOC ${ }^{16}$. Dichas agudizaciones influyen en la morbilidad, en la calidad de vida, incrementan la mortalidad, generan un alto consumo de recursos y desde el punto de vista pronóstico, el número de agudizaciones experimentadas 
en el pasado es uno de los mejores predictores de riesgo para presentarlas en el futuro ${ }^{16,44-46}$. El 50-70\% de las agudizaciones se deben a infección bronquial bacteriana o viral, sin que los criterios clínicos permitan diferenciar la causa ${ }^{47}$. La neumonía adquirida en la comunidad es un proceso de alta prevalencia en la población general y los datos actuales apuntan a una mayor frecuencia en la población con EPOC, comunicándose 55 casos por cada 1000 pacientes y año, cifras mucho más altas que las de la población general ${ }^{48}$. En nuestro medio el neumococo seguido del $H$. influenzae son los principales agentes responsables, si bien en los últimos tiempos cobran protagonismo bacterias atípicas (legionella, chlamydia pneumoniae). Un estudio reciente confirma el peor pronóstico de los pacientes con EPOC y neumonía; la mortalidad llega al 20\% frente al 5,8\% cuando la exacerbación se debe a otra causa ${ }^{49}$.

Reducir la patología infecciosa en el paciente con EPOC supondría una modificación sustancial de su historia natural. Para ello contamos con tres medidas: a) inmunización antigripal anual y antineumocócica, b) incluir en pacientes de riesgo en fase estable, fármacos que han demostrado reducir las agudizaciones (tiotropo, beta-2 agonistas de acción larga, glucocorticoides inhalados y roflumilast en casos seleccionados), y c) ante la sospecha de infección bacteriana elegir los antimicrobianos que tengan mayor capacidad de erradicación bacteriana $^{16,50}$.

\section{Ansiedad y depresión en la EPOC}

La limitación progresiva al flujo aéreo de la EPOC se traduce en disnea que afecta de forma paulatina la actividad física de los pacientes con afectación secundaria de su estado psicológico y social, lo que influye desfavorablemente en su entorno personal, profesional y laboral, con lo que aumenta de forma considerable la probabilidad de dependencia y aislamiento social. En este contexto, no es raro que hasta el $50 \%$ de los pacientes presenten trastornos psicológicos, estimándose una prevalencia del $15 \%$ para la ansiedad generalizada y del $25 \%$ para la depresión ${ }^{16,51,52}$. Van Manen et al concluyen que el riesgo de depresión en el paciente con EPOC es 2,5 veces superior al de la población general, después de ajustar por variables demográficas y comorbilidad ${ }^{51}$. Además la depresión y la ansiedad en la EPOC se asocia a peor pronóstico siendo predictora de mortalidad y de mayor estancia hospitalaria ${ }^{16}$.

El tratamiento farmacológico de estos pacientes debe ser similar al de la población general, pues apenas existen estudios de intervención en estas situaciones clínicas. Al utilizar fármacos ansiolíticos las benzodiacepinas se deben manejar con precaución por el efecto depresor de la función respiratoria, considerándose de elección la buspirona porque no presenta efectos adversos respiratorios ${ }^{53}$. Entre los fármacos antidepresivos, los inhibidores de la recaptación de serotonina y los inhibidores de recaptación de serotonina y noradrelina son los de elección por su nulo efecto sobre la función respiratoria, buena tolerancia y pocos efectos secundarios ${ }^{16}$.

\section{Otras comorbilidades}

Los pacientes con EPOC presentan una asociación mayor de la esperada de diabetes y síndrome metabólico. El estudio ECCO recoge el antecendente de diabetes en un 30\% de los pacientes ${ }^{13}$ y se describe una prevalencia de síndrome metabólico próximo al $15 \%$ en los EPOC europeos ${ }^{54}$. Probablemente el nexo patogénico de estas entidades resida en la respuesta inflamatoria local y sistémica que está íntimamente ligada al desarrollo de resistencia insulínica e hiperglucemia.

En los pacientes con EPOC concurren ciertas patologías digestivas con mayor prevalencia que en la población general, entre las que destacan por número e implicaciones clínicas la enfermedad ulcerosa péptica, la enfermedad por reflujo gastroesofágico y determinadas etiologías de hepatopatía crónica. La implicación de la colonización gástrica por Helicobacter pilory en la patogenia de la EPOC se fundamenta en una serie de estudios de seroprevalencia que han confirmado la conocida comorbilidad entre úlcera péptica y bronquitis crónica ${ }^{55}$. También en estos pacientes es más prevalente el reflujo gastroesofágico clínicamente significativo y su presencia ha sido involucrada en la frecuencia y severidad de sus exacerbaciones $^{56}$. Entre el 1 y el $2 \%$ de todos los casos de enfisema pulmonar se asocia a déficit de alfa-1-antitripsina, enfermedad sistémica hereditaria que desarrolla enfisema precoz y cirrosis hepática. La SEPAR, siguiendo las guías americanas y europeas, recomienda determinar al menos en una ocasión el nivel sérico de alfa-1-antitripsina en todo individuo diagnosticado de $\mathrm{EPOC}^{57}$.

En definitiva la EPOC es un proceso muy complejo que trasciende el ámbito estrictamente pulmonar y se asocia con un elevado número de enfermedades que afectan a distintos órganos. Dos son los factores más relevantes que explican este comportamiento: a) el tabaco, principal factor de riesgo para desarrollar EPOC, coincide como agente etiológico fundamental en muchos otros procesos, y b) la capacidad del tabaco como potente inductor de reacción inflamatoria, por agresión celular directa y mediante el incremento de mediadores de inflamación, que revela a la EPOC como una verdadera enfermedad sistémica que comparte muchas características comunes con una gran variedad de enfermedades.

\section{Bibliografía}

1. Mathers CD. The global burden of disease: 2004 update. Ginebra, Organización Mundial de la Salud, 2008.

2. Barnes PJ (2007) Chronic obstructive pulmonary disease: A growing but neglected global epidemic. PLoS Med 4(5): e112. doi:10.1371/journal.

3. Halbert RJ, Natoli JL, Gano A, Badamgarav E, Buist AS, Mannino DM. Global burden of COPD: systematic review and meta-analysis. Eur Respir J. 2006; 28: 523-32.

4. Ministerio de Sanidad y Política social. Estrategia en EPOC del Sistema Nacional de Salud. Centro de Publicaciones Ministerio de Sanidad y Consumo: Madrid, 2009. 
5. Sobradillo-Peña V, Miravitlles M, Jiménez CA, Gabriel R, Viejo JL, Masa JF, et al.. Estudio Epidemiológico de la Enfermedad Pulmonar Obstructiva Crónica en España (IBERPOC): Prevalencia de síntomas respiratorios crónicos y limitación del flujo aéreo. Arch Bronconeumol. 1999; 35: 159-66.

6. Soriano JB, Miravitlles M, Borderías L, Duran-Tauleria E, García Río F, Martínez J, Montemayor T et al. Diferencias geográficas en la prevalencia de EPOC en España: relación con hábito tabáquico, tasas de mortalidad y otros determinantes. Arch Bronconeumol 2010; 46: 522-30.

7. Miravitlles M, Murio C, Guerrero T, Gisbert R on behalf of the DAFNE study group. Costs of chronic bronchitis and COPD. A one year follow-up study. Chest. 2003; 123: 784-91.

8. Anguita Sánchez M, Crespo Leiro MG, De Teresa Galván E, et al. Prevalencia de la insuficiencia cardiaca en la población general española mayor de 45 años. Estudio PRICE. Rev Esp Cardiol 2008; 61: 1041-9.

9. Montes Santiago J. Arte y riesgo cardiovascular. Una gañería de cifras y figuras. S\&H Meicla Science: Madrid, 2010.

10. J. Montes-Santiago J, R. Guijarro-Merino R, San Román-Terán C, Monreal M. Hospitalizaciones por insuficiencia cardíaca y epoc en españa. concomitancias y diferencias. Rev Clin Esp 2011 (Supl. Esp): 211:14.

11. Montero Pérez-Barquero M, Conthe Gutiérrez P, Román Sánchez P, Forteza-Rey J; Grupo de Trabajo de Insuficiencia Cardiaca de la Sociedad Española de Medicina Interna (estudio SEMI-IC).Comorbilidad de los pacientes ingresados por Insuficiencia Cardiaca en los servicios de Medicina Interna. Rev Clin Esp. 2010; 210:149-58.

12. Jiménez Muñoz AB. Epidemiología de la EPOC e IC. Aspectos pronósticos. En: Méndez Bailón M, de Miguel Díaz J, Comín Colet J, coord. EPOC e Insuficiencia Cardiaca: Una combinación frecuente y a menudo ignorada. Madrid: Gráficas Prado \& Robepra SL; 2008, 7-22.

13. Almagro P, López García F, Cabrera FJ, Montero L, Morchón D, Díez J, de la Iglesia F, Roca FB, Fernández-Ruiz M, Castiella J, Zubillaga E, Recio J, Soriano JB; Grupo EPOC de la Sociedad Española de Medicina Interna. Estudio de las comorbilidades en pacientes hospitalizados debido a enfermedad pulmonar obstructiva crónica atendios en servicios de Medicina Interna. Estudio ECCO. Rev Clin Esp. 2010; 210:101-8

14. Zapatero Gaviria A, Barba Martín R y Grupo Gestión FEMI. Estudio de un millón de altas hospitalarias en Medicina Interna. Disponible en: http://www.fesemi.org (consultado 04/12/2010).

15. Boixeda R, Almagro P, Díez J, Custardoy J, López García F, San Román Terán C, Recio J, Soriano JB; en representación del grupo EPOC de la Sociedad Española de Medicina Interna. Características clínicas y tratamiento de los pacientes ancianos hospitalizados por descompensación de enfermedad pulmonar obstructiva crónica en los servicios de Medicina Interna espanoles. Estudio ECCO. Med Clin (Barc) 2011; ( Nov 24, Epub ahead of print].

16. Global initiative for Chronic Obstructive Lung Disease. Global strategy for the diagnosis, management, and prevention of chronic obstructive pulmonary disease - 2011 updated [consulta 26 de enero de 2012]. Disponible en: www.golcopd.com.

17. O'Shaughnessy TC, Ansari TW, Barnes NC, Jeffery PK. Inflammation in bronchial biopsies of subjects with chronic bronchitis: inverse relationship of CD8+ T lymphocytes with FEV 1 . Am J Respir Crit Care Med. 1997; 155: 852-7.

18. Shapiro SD. Elastolytic metalloproteinases produced by human mononuclear phagocytes. Potential roles in destructive lung disease. Am J Respir Crit Care Med. 1994; 150 (6 Pt 2): S160-4

19. Agustí A. Systemics effects of chronic obstructive pulmonary disease: what we know and what we don't know (but should). Proc Am Thorac Soc. 2007; 4: 522-5

20. Decramer M, De Benedetto F, Del Ponte A, Marinari S. Systemic effects of COPD. Respir Med. 2005; 99 (Supl. B): S3-10.

21. Agustí A, Soriano JB. COPD as a systemic disease. COPD. 2008; 5: 133-8.

22. Cazzola M, Matera MG, Rogliani P, Page C. Treating systemic effects of COPD. Trends Pharmacol Sci. 2007; 28: 544-50.

23. Decramer M, Rennard S, Troosters T, Mapel DW, Giardino N, Mannino D, et al. COPD as a lung disease with systemic consequences-clinical impact, mechanism and potential for early intervention. COPD. 2008; 5: 235-56.

24. Calverley PM, Anderson JA, Celli B, Ferguson GT, Jenkins C, Jones PW, et al. Salmeterol and fluticasone propionate and survival in chronic obstructive pulmonary disease. N Engl J Med. 2007; 356: 775-89.

25. Bale G, Martínez-Camblor P, Burge PS, Soriano JB. Long-term mortality follow-up of the ISOLDE participants: Causes of death Turing 13 years alter trial completion. Respir Med. 2008; 102: 1468-72.

26. Charlson ME, Szatrowski TP, Peterson J, Gold J. Validation of a combined comorbidity index. J Clin Epidemiol. 1994; 47: 1245-51.

27. Almagro P, Calvo E, Ochoa de Echagüen A, Barreiro B, Quintana S, Heredia JL e tal. Mortality after hospitalization for COPD. Chest. 2002; 121: 1441-8

28. Sunyer J, Ulrik CS. Level of FEV1 as a predictor of all-cause and cardiovascular mortality: an effect beyond smoking and physical fitness. Eur Respir J. 2005; 25: 587-8.
29. Soriano JB, Maier WC, Egger P, Visick G, Thakrar B, Sykes J et al. Recent trends in physician diagnosed COPD in women and men in the UK. Thorax. 2000; 55: 789-94.

30. Sidney S, Sorel M, Quesenberry CP Jr, DeLuise C, Lanes S, Eisner MD. COPD and incident cardiovascular disease hospitalizations and mortality: Kaiser Permanente Medical Care. Chest. 2005; 128(4): 2068-75.

31. Holgin F, Folch E, Redd SC, Mannino DM. Comorbidity and mortality in COPD-related hospitalizations in the US, 1979 to 2001. Chest. 2005; 128(4): 2005-11.

32. Huiart L, Ernst P, Suissa S. Cardiovascular morbidity and mortality in COPD. Chest. 2005; 128(4): 2640-6.

33. Lobby $\mathrm{P}$, Ridker PM. Inflammation and atherosclerosis: role of $\mathrm{C}$-reactive protein in risk assessment. Am Med J. 2004; 116Suppl6A: 9S-16S.

34. Sin DD, Man SF. Why are patients with chronic obstructive pulmonary disease at increased risk of cardiovascular diseases? The potential role of systemic inflammation in chronic obstructive pulmonary disease. Circulation. 2003; 107: 1514-9.

35. Salpeter S, Ormiston T, Salpeter E. Cardioselective beta-blockers for chronic obstructive pulmonary disease. Cochrane database of systematic reviews 2005:CD003566.

36. Render ML, Weinstein AS, Blaustein AS. Left ventricular dysfunction in deteriorating patients with chronic obstructive pulmonary disease. Chest. 1995; 107: 162-8.

37. Truelsen T, Prescott E, Lange P, Schnohr P, Boysen G. Lung function and risk of a fatal and non-fatal stroke. The Copenhagen City Heart Study. Int J Epidemiol. 2001; 30: $145-51$.

38. Hozawa A, Billings JL, Sharar E, Ohira T, Rosamond WD, Folsom AR. Lung function and ischemic stroke incidence: the Atherosclerosis Risk in Communities Study (ARIC). Chest. 2006; 130: 1642-9.

39. Prescott SM, Richards KL, Tikoff G, Amstrong TD Jr, Shigeoka JW. Venous thromboembolism in descompesated chronic obstructive pulmonary disease. A prospective study. Am Rev Respir Dis. 1981; 123: 32-6.

40. Tillie-Leblond I, Marquette C, Perez T, Scherpereel A, Zanetti C, Tonel AB et al. Pulmonary embolism in patients with unexplained exacerbation of chronic obstructive pulmonary disease: prevalence and risk factors. Ann Intern Med. 2006; 144: 390-6.

41. Mannino DM, Watt G, Hole D, Gillis C, Hart C, McConnachie A et al. The natural history of chronic obstructive pulmonary disease. Eur Respir J. 2006; 27: 627-43.

42. Soriano JB, Visick GT, Muellerova H, Payvandi N, Hansell AL. Patterns of comorbidities in newly diagnosed COPD and asthma in primary care. Chest. 2005; 128: 2099-107.

43. Miravitlles M. Exacerbations of chronic obstructive pulmonary disease: when are bacterial important? Eur Respir J. 2002; 20: 9s-19s.

44. Groenewegen KH, Schols AM, Wouters EF. Mortality and mortality-related factors after hospitalization for acute exacerbation of COPD. Chest. 2003; 124: 459-67.

45. Hilleman DE, Dewan N, Malesker M, Friedman M. Pharmacoeconomic evaluation of COPD. Chest. 2000; 118: 1278-85.

46. Miravitlles M, Guerrero T, Mayordomo C, Sánchez-Agudo L, Nicolau F, Segú JL on Behalf of the EOLO Group. Factors associated with increased risk of exacerbation and hospital admission in a cohort of ambulatory COPD patients: a multiple logistic regression analysis. Respiration. 2000; 67: 495-501.

47. Sapey E, Stockley RA. COPD exacerbations. Aetiology. Thorax 2006; 61: 250-8.

48. Merino-Sánchez M, Alfageme-Michavilla I, Reyes-Nuñez N, Lima-Alvarez J. Prognosis in patients with pneumonia and chronic obstructive pulmonary disease. Arch Bronconeumol. 2005; 41: 607-11.

49. Steer J, Norman EM, Afolabi OA, Gibson GJ, Bourke SC. Dyspnoea severity and pneumonia as predictors of in-hospital mortality and early readmission in acute exacerbation of COPD. Thorax. 2012; 67: 117-21.

50. Miravitlles M, Torres A. Antibiotics in exacerbations of COPD: lessons from the past. Eur Respir J. 2004; 24: 896-7.

51. Van Manen JG, Bindels PJE, Dekker FW, ljzermans CJ, Van Der Zee JS, Schadé E. Risk of depresión in patients with chronic obstructive pulmonary disease and its determinants. Thorax. 2002; 57: 412-6.

52. Mikkelsen RL, Middeloble T, Pisinger C, Stage KB. Anxiety and depression in patients with chronic obstructive pulmonary disease (COPD). A review. Nord J Psychiatry. 2004; 58: 65-7.

53. Argyropoulou P, Patakas D, Koukou A, Vasiliadis P, Georgopoulos D. Buspirone effect on breathlessness and exercise performance in patients with chronic obstructive pulmonary disease. Respiration. 1993; 60: 216-20.

54. Hu G, Qiao A, Tuomilehto J, Balkau B, Borch-Johnsen K, Pyorala K, for the DECODE Study Group. Prevalence of the metabolic syndrome and its relations to all-cause and cardiovascular mortality in non-diabetic European men and women. Arch Intern Med. 2004; 164: 1066-76.

55. Caselli M, Zaffoni E, Ruina M, Sartori S, Trevisani L, Ciaccia A et al. Helicobacter pilory and chronic bronchitis. Scand J Gastroenterol. 1999; 34: 828-30.

56. Rascon-Aguilar IE, Pamer M, Wludyka P, Cury J, Coultas D, Lambiase LR et al. Role of gastroesophageal reflux symptoms in patients with COPD. Chest. 2006; 130: 1096-101. 